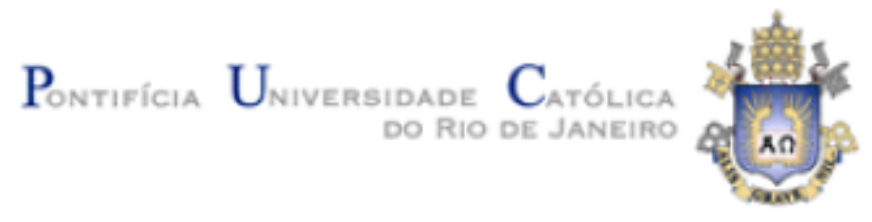

Pedro Massao Favaro Nakashima

\title{
Análise empírica das intervenções cambiais do Banco Central do Brasil usando dados de alta frequência
}

Dissertação apresentada como requisito parcial para obtenção do grau de Mestre pelo Programa de PósGraduação em Economia do Departamento de Economia da PUC-Rio.

Orientador: Prof. Márcio Gomes Pinto Garcia Coorientador: Prof. Marcelo Cunha Medeiros

Rio de janeiro

Abril de 2012 


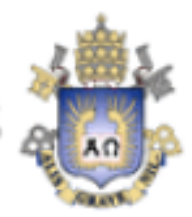

Pedro Massao Favaro Nakashima

\begin{abstract}
Análise empírica das intervenções cambiais do Banco Central do Brasil usando dados de alta frequência
\end{abstract}

Dissertação de Mestrado Dissertação apresentada como requisito parcial para obtenção do grau de Mestre pelo Programa de PósGraduação em Economia do Departamento de Economia da PUC-Rio. Aprovada pela Comissão Examinadora abaixo assinada.

Prof. Márcio Gomes Pinto Garcia

Orientador

Departamento de Economia - Puc-Rio

Prof. Marcelo Cunha Medeiros Coorientador Departamento de Economia - Puc-Rio

Prof. Carlos Viana de Carvalho Departamento de Economia - Puc-Rio

Wenersamy Ramos de Alcântara Banco Central do Brasil

Profa. Mônica Herz

Coordenadora Setorial do Centro de Ciências Sociais 
Todos os direitos reservados. É proibida a reprodução total ou parcial do trabalho sem autorização da universidade, do autor e do orientador.

\section{Pedro Massao Favaro Nakashima}

Graduou-se em Ciências Econômicas pela Universidade Federal de Mato Grosso do Sul. Cursou Mestrado em Economia entre 2010 e 2012.

Ficha Catalográfica

Nakashima, Pedro Massao Favaro

Análise empírica das intervenções cambiais do Banco Central do Brasil usando dados de alta frequência / Pedro Massao Favaro Nakashima ; orientador: Márcio Gomes Pinto Garcia ; coorientador: Marcelo Cunha Medeiros. - 2012.

110 f. : il. (color.) ; $30 \mathrm{~cm}$

Dissertação (mestrado)-Pontifícia Universidade Católica do Rio de Janeiro, Departamento de Economia, 2012.

Inclui bibliografia

CDD: 330 
Aos meus pais, José e Jandira, por tudo. 


\section{Agradecimentos}

Aos meus pais, José Tsuyoshi Nakashima e Jandira Bonfietti Favaro Nakashima, por todo o amor e apoio durante todos esses anos.

Aos meus orientadores, Professores Márcio Garcia e Marcelo Medeiros, que em muito contribuíram para essa dissertação e também para a minha formação.

Ao Professor Carlos Viana de Carvalho e ao Wenersamy Ramos de Alcântara, integrantes da Comissão Avaliadora, que, com suas críticas e sugestões, adicionaram valor a esta dissertação.

A todos os professores e funcionários do Departamento de Economia da Puc-Rio, por construírem o melhor Departamento de Economia do Brasil.

À BM\&FBovespa e ao Banco Central do Brasil, pelo fornecimento dos dados utilizados nessa dissertação.

A todos os colegas do mestrado, pelas críticas e sugestões dadas.

À CAPES e à PUC-Rio, pelo apoio financeiro concedido. 


\section{Resumo}

Nakashima, Pedro Massao Favaro; Garcia, Márcio Gomes Pinto; Medeiros, Marcelo Cunha. Análise empírica das intervenções cambiais do Banco Central do Brasil usando dados de alta frequência. Rio de Janeiro, 2012. 104 p. Dissertação de Mestrado - Departamento de Economia, Pontifícia Universidade Católica do Rio de Janeiro.

O objetivo desta dissertação é investigar a existência de retornos anormais no contrato de dólar futuro de primeiro vencimento nos momentos próximos à realização dos leilões de câmbio pelo Banco Central do Brasil. Em respondendo positivamente à primeira indagação, avaliamos a persistência de tais retornos que, em última instância, descreverá o quão rápido novas informações são difundidas e incorporadas aos preços. Para isso, aplicamos os arcabouços de estudos de eventos e microestrutura do mercado de câmbio aos dados de alta frequência obtidos junto à BM\&FBovespa e ao BC. A disponibilidade dos dados dos negócios permite que se utilize da variável de fluxo de ordem e, assim, se corrijam potenciais problemas relacionados a viés de variável omitida, não considerados na literatura sobre intervenções dos bancos centrais. Encontram-se retornos anormais estatisticamente positivos nos minutos que seguem à abertura de um leilão e negativos nos minutos seguintes ao fechamento do mesmo. Adicionalmente, as evidências mostram que o horário do leilão pouco afeta os resultados.

\section{Palavras-chave}

Intervenções cambiais do Banco Central; microestrutura de mercado; estudos de eventos; mercado de câmbio brasileiro. 


\section{Abstract}

Nakashima, Pedro Massao Favaro; Garcia, Márcio Gomes Pinto (Advisor); Medeiros, Marcelo Cunha (Co-advisor). Empirical analysis of Brazilian central bank's foreign exchange interventions using high frequency data. Rio de Janeiro, 2012. 104 p. MSc Dissertation - Departamento de Economia, Pontifícia Universidade Católica do Rio de Janeiro.

The goal of this dissertation is to investigate the existence of abnormal returns in U.S. dollar futures contract maturing in the first moments near to the realization of exchange auctions by the Central Bank of Brazil. On the occasion of a positive response to the first question, we evaluated the persistence of these returns that ultimately describe how fast new information is disseminated and incorporated into prices. For this, we apply the frameworks of Event Studies and Microstructure of the exchange market to high-frequency data obtained from the BM\&FBovespa and the CB. The availability of transactions data allows the use of variable order flow and thus to correct potential problems related to omitted variable bias, not considered in the literature on Central Bank intervention. We found statistically positive abnormal returns in the minutes following the opening of an auction and negative in the minutes following the closure of the same event. Additionally, the evidence points to the direction that, among the times chosen by the monetary authority in the database, there is no statistically significant difference with respect to the effects of such events on the movements in the exchange rate on intraday basis.

\section{Keywords}

Foreign Exchange intervention by the Central Bank; Market microstructure; event studies; Brazilian FX markets. 


\section{Sumário}

1. Introdução 12

2. Revisão de literatura 19

3. Base de dados 24

4. Intervenções cambiais do BC 26

5. Estratégia empírica 30

5.1. Definição de evento no contexto dos leilões 31

5.2. Agregação temporal 33

5.2.1. Frequência de agregação 39

5.3. Arcabouço de Estudos de eventos 39

5.3.1. Descrição do método 40

5.3.2. Regressão por dummies 44

6. Resultados empíricos $\quad 47$

6.1. Resultados empíricos, agregação de 1 minuto em torno da abertura 50

6.2. Resultados empíricos, agregação de 1 minuto em torno do fechamento 59

6.3. Resultados empíricos, agregação de 20 segundos em torno da abertura 
7.1. THC, agregação de 1 minuto em torno da abertura 73

7.2. THC, agregação de 1 minuto em torno do fechamento 78

8. Extensões 84

8.1. Comparação com outros resultados encontrados na literatura 84

8.2. O horário da intervenção importa? 86

8.3. High frequency trading (HFT) 93

8.4. Questões normativas e oportunidades de investimento 98

9. Validade interna e externa 100

10. Conclusão 103

$\begin{array}{ll}\text { Referências bibliográficas } & 106\end{array}$

Apêndice: construção da base de dados 109 


\section{Lista de figuras}

Figura 1 - Volume e fluxo de ordem, médios, agr. 1min (ab) 14

Figura 2 - Número de leilões por dia 22

Figura 3 - Taxa de câmbio diária no período amostral 23

Figura 4 - Linha do tempo de estudos de eventos 40

Figura 5 - Absorção do impacto pelo coeficiente do F.O. 57

Figura 6 - Elevação do impacto do F.O. (nos retornos) na abertura - modelo M5

Figura 7 - Retornos anormais e impacto do f.o.: modelos M7 a M10 65

Figura 8 - Elevação do impacto do F.O. (nos retornos) na abertura - modelo M13

Figura 9 - Testes de significância para soma dos coeficientes modelos M2 e M4

Figura 10 - Testes de significância para soma dos coeficientes modelos M3 e M5

Figura 11 - Testes de significância para soma dos coeficientes modelos M7 e M9

Figura 12 - Testes de significância para soma dos coeficientes modelos M8 e M10

Figura 13 - Número de negócios e leilões por faixa de horário

Figura 14 - Evolução da taxa de câmbio no dia 6 de maio de 2010 


\section{Lista de tabelas}

Tabela 1 - Estimativas dos modelos para os retornos percentuais de $1 \mathrm{~min}(\mathrm{ab})$

Tabela 2 - Estimativas dos modelos para os retornos percentuais de $1 \mathrm{~min}(\mathrm{fe})$

Tabela 3 - Estimativas dos modelos para os retornos percentuais de 20s (ab)

Tabela 4 - Testes progressivos de hipóteses conjuntas, agr $1 \mathrm{~min}$ (ab)

Tabela 5 - Testes progressivos de hipóteses conjuntas, agr $1 \mathrm{~min}$ (fe)

Tabela 6 - Impacto do f.o. nos retornos por faixa de horário

Tabela 7 - Influência do horário no impacto dos leilões nos retornos

Tabela 8 - Influência da NYSE no modelo m3

Tabela 9 - Resultados do modelo m3 modificado, dia 6 de maio de 2010 